\title{
Investigation of Inverse Magnus Effect by Partial Circulation Control Elements: Experimental Design
}

\author{
Acar Celik $^{1}$, Sercan Acarer', Ian Jacobi², Beni Cukurel² \\ ${ }^{1}$ Izmir Katip Celebi University \\ Izmir, Turkey \\ Y180228005@ogr.ikcu.edu.tr; sercan.acarer@ikcu.edu.tr \\ ${ }^{2}$ Technion - Israel Institute of Technology \\ Haifa, Israel \\ ijacobi@technion.ac.il
}

\begin{abstract}
The inverse Magnus effect is a special physical state that can only be achieved within certain ranges of the Reynolds number and the spin ratio. Numerical studies and particularly, special experimental setups are required for its investigation. In this study, an experimental design idea that will enable the mentioned physical phenomenon to be analyzed on a specific geometry is presented. By compiling similar studies in the past years, the innovations made are emphasized. All the elements on the assembly whose design has been completed are detailed and its advantages and disadvantages are mentioned. It is predicted that the system with the partially rotating cylinders planned to be established in the near future will be a guide in numerical studies and will create important outputs in the use of the Magnus effect on airfoils in aviation.
\end{abstract}

Keywords: experiment, Magnus, PIV, design

\section{Introduction}

The Magnus effect is defined as a physical phenomenon that causes objects moving in a flow environment by rotating around their own axis, leaving their basic orbit to continue their motion. This effect was first observed in 1671 by G.T. Walker, but it was added to the literature with the work of G. Magnus [1]. Lafay [2] then carried out the first experimental studies in detail.

An unexpected external force occurs on a rotating body released into a flow medium. The rotating body directs the flow of air in the direction it rotates, changing the pressure distribution around it according to Bernoulli's principle, causing this non-symmetrical distribution on the body to increase the pressure difference in one direction. The magnitude of the force on the body is directly proportional to the interaction between the tangential velocity on the surface of the object and the main flow velocity. The basic principle of the Magnus effect is given in Fig. 1.

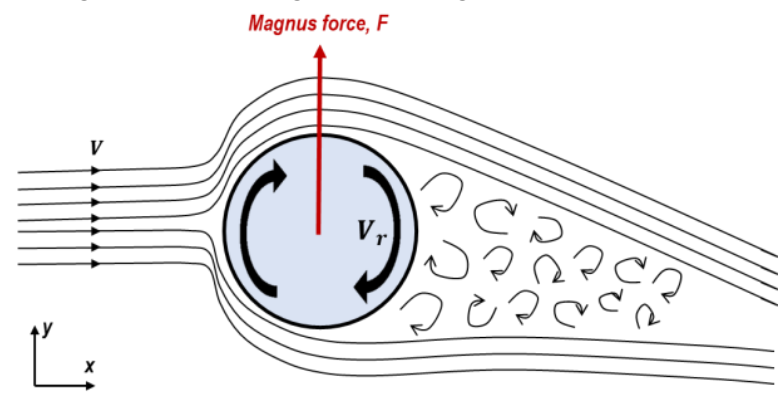

Fig. 1: Magnus effect

In the past years, contrary to expectations, a second force has been proposed which has the opposite direction compared with the force in the Magnus effect. As a result of numerical and experimental studies, it has been reported that the direction of the flow changes at certain range of Reynolds number $(\mathrm{Re})$ and spin ratio $(\mathrm{Vr} / \mathrm{V})$ and the resulting force is in the opposite direction to what is expected [1,3,4,5]. This change is called the inverse Magnus effect. The basic formation mechanism of the inverse Magnus effect is related to the local Reynolds number acting at every point around the body. 


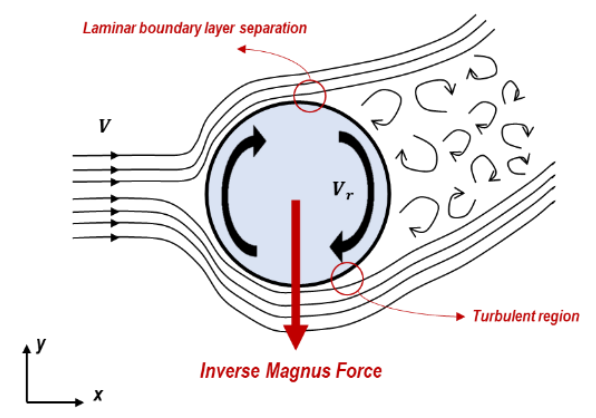

Fig. 2: Inverse Magnus effect

In the mechanism seen in Fig. 2, the local Reynolds number decreases in a region at the top of the cylinder. For this reason, the separation of the flow is much earlier and occurs as a laminar. In contrast to the upper part, the Reynolds number is remarkably high in the lower part and a turbulent boundary layer separation is observed. Turbulence directs the flow upwards with momentum transfer and the lift force on the body is in the opposite direction.

Swanson [1] compiled studies involving the physics of a rotating cylinder released into a flow medium. He made inferences by adding his own experimental studies to the data he collected. A broad-spectrum investigation was carried out with the comparisons obtained. Fig. 3 gives the variation of the lift force depending on the spin ratio from that work.
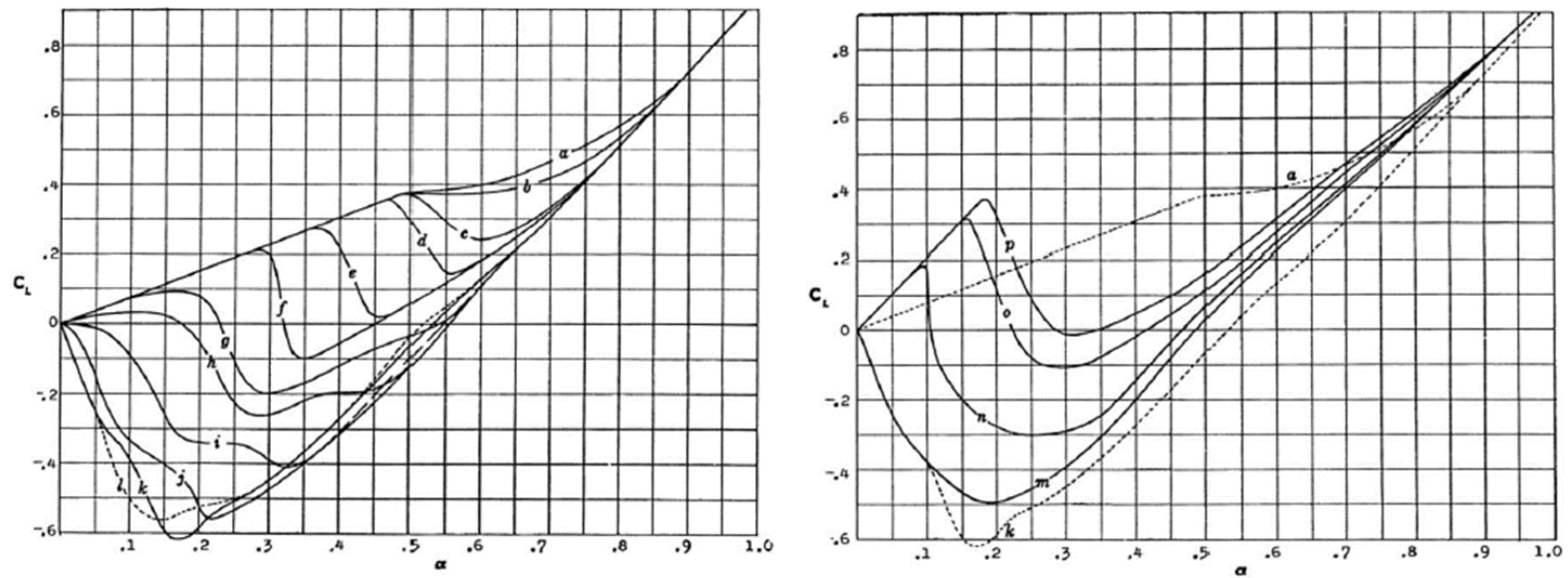

Fig. 3: Lift coefficient change over spin ratio (a-f: $R e=3.58 \mathrm{E}+04-12.8 \mathrm{E}+04, \mathrm{~g}-\mathrm{m}: \mathrm{Re}=15.2 \mathrm{E}+04-36.5 \mathrm{E}+04, \mathrm{n}-\mathrm{p}: \mathrm{Re}=$ $42 \mathrm{E}+04-50.1 \mathrm{E}+04)[1]$

As a result of the study, it was stated that the reverse Magnus effect was observed in the experiments with different rotation ratios with Reynolds values between 99000 and 501000. This effect is currently being studied in some experimental studies. Kray et al. [6] performed force and flow field measurements on a sphere in a wide spectrum of Reynolds number and spin ratio. In his studies, he emphasized the effect of changing parameters on the location and type of flow separation. He reported that the negative Magnus effect appears with the gradually increasing spin ratios. In another study, Kim et al. [7] investigated the Magnus effect with particle imaging velocity measurement on a similar sphere. Considering the characteristics of the boundary layer separations on the sphere, it has been claimed that the inverse Magnus effect is dependent on these dynamics. It has been shown that the shear layer instability due to the transition to turbulence especially in the separation of the flow formed at the bottom of the cylinder causes the flow to reattach to the surface. All studies given include an experimental method. Experimental studies are of great importance, especially in the verification of existing numerical methods on the aerodynamics of rotating bodies. 
In this study, a design of an experimental setup where inverse Magnus and Magnus effects can be created by applying similar scenarios on a unique cylinder geometry is presented. The details and mechanism of the design are explained in pictures and comparisons.

\section{Experimental Design}

In this work, the efforts were given to design the experimental setup, considering the novel and compelling geometry. geometry. Principal component of the test rig is given with Fig. 4.

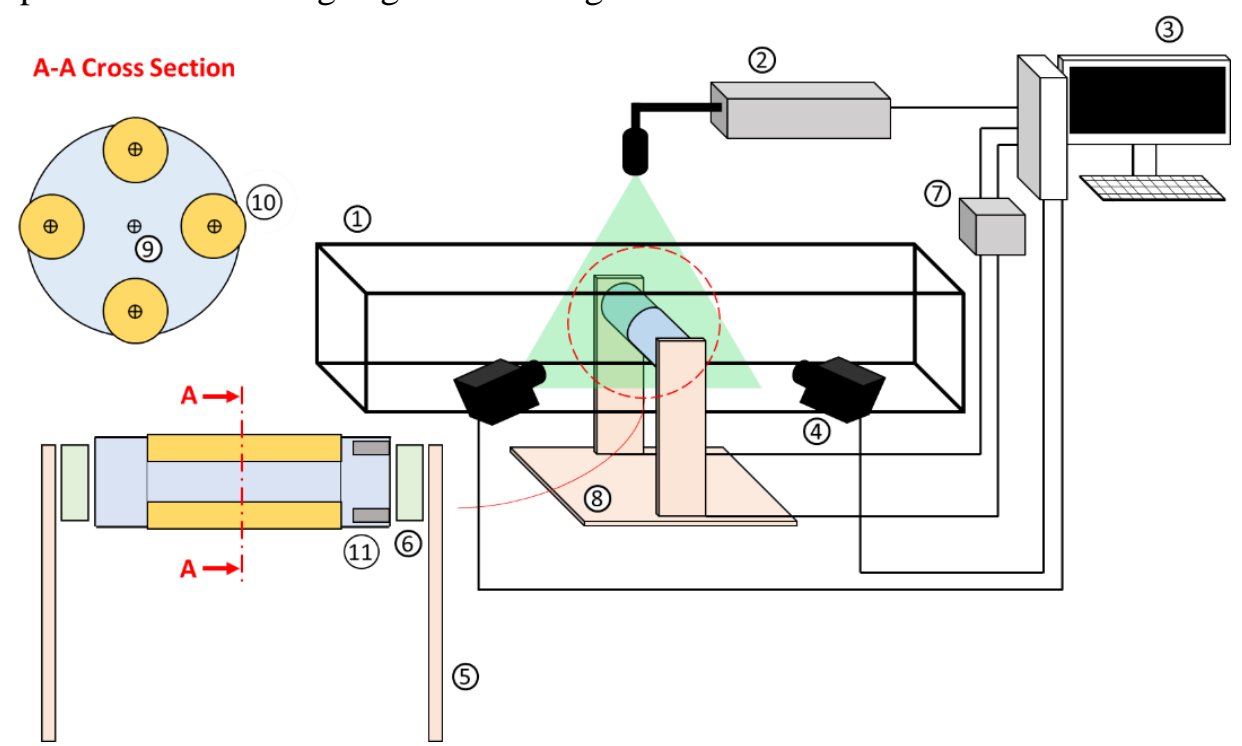

Fig. 4: Elements of the experimental setup (1: Water tunnel test section, 2: Laser, 3: Computer, 4: High speed camera, 5: Force balance rod, 6: Load cell, 7: Amplifier, 8: Balancing table, 9: Stator, 10: Rotor (actuator), 11: DC-Motor)

The basis of the design was inspired from the study of Modi [8]. The main part of the test section has two components: stator and rotor. It was planned to change the scenarios by using 4 actuators around the stator, also adding the 5th degree of freedom by the angular configuration of stator. Rotors are driven by 4 DC-motors individually. Two types of measurements are aimed to be performed. Primarily, unique force balance design including two 3-axis load cells was completed to measure the lift and the drag forces. During the experiments, data read signals is amplified to be monitored on the computer. At the same time, PIV measurements are implemented on the test section. PIV system contains two high speed cameras, one class 4 laser, computer, and the software for post-processing. Obtained force data and the flow visualization elements are intended to be combined and be interpreted together. The most crucial point will be observing the inverse Magnus effect and understanding its relationship with the flow characteristics.

Whole test rig is immersed in a water tunnel. Therefore, various sealing solutions were considered. Electronic equipment, such as, motors and encoders were kept apart from water as far as possible. Waterproof bearings were placed rotor ends to prevent leakage inside the whole system. Fig. 5b and Fig. 5c indicates the visualization of the test rig. In Fig. $5 \mathrm{a}$, cross section of the whole system is represented. Both ends of the test mechanism has the identical components in a symmetrical manner. From left to right (see Fig. 5a), force balance rod, load cell, flange among the load cell to the motor housing, motor housing and the motors, coupling housing and the couplings, bearings and shaft sealings may be seen, respectively. Motors drive the rotors by the assembly of Oldham type couplings. Shaft seal and sealed bearings intercept the potential danger by water. Regarding the test section assembly to the acrylic panels of the water tunnel, fabric penetration sealing elements are chosen. In this way, force balance measurements are aimed to be performed properly by providing six degrees of freedom to the whole system. Rotors are located inside the spaces opened onto the stator. The gap between rotor and stator were selected as $200 \mu \mathrm{m}$, where the motor power and the torque were calculated by. The measurement uncertainty of the lift and drag is calculated as $\pm 0.5 \%$. For that calculation, the sensitivity of load cell and the encoder of the motor were 
considered. Eventually, each part was design in the frame of manufacturability and feasibility. Most of the components are already collected and the assembly process will be initiated in the near future.

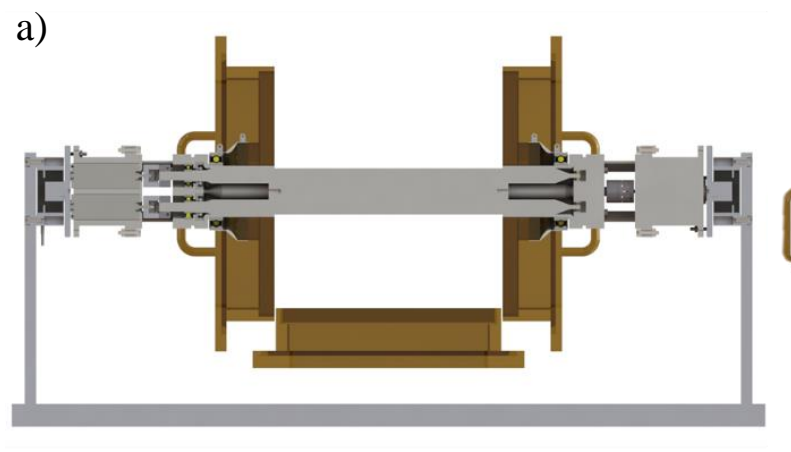

b)
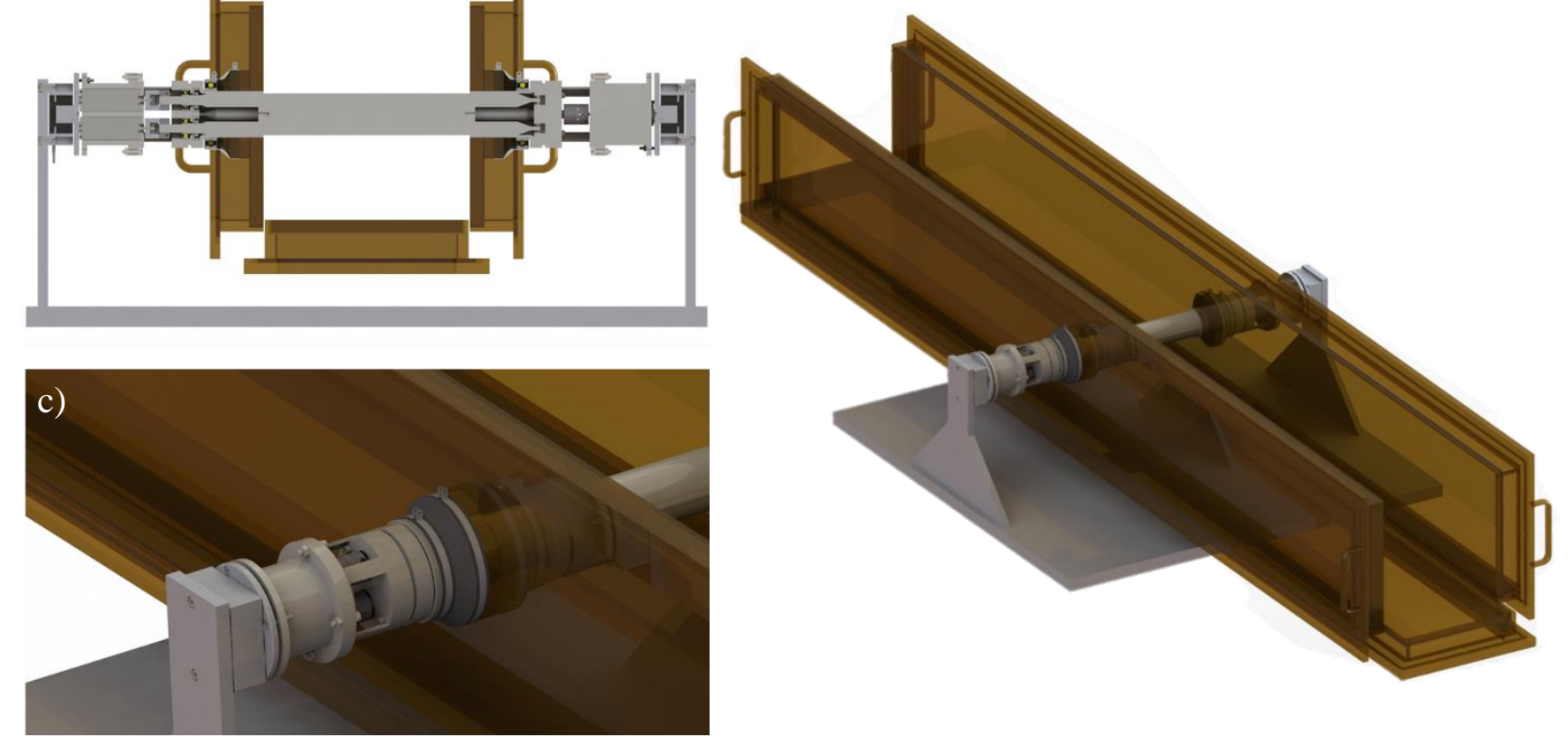

Fig. 5: a) Cross section of the system, b) Experimental test section, c) Detailed look of sealing and driver section

\section{Conclusion}

Problems through flow over a cylinder has always become the benchmark cases in the past of the external fluid dynamics. That point of view is of great inspiration to this work, choosing the basic sort of geometry. The introduced experimental design ensures a novel way to generate circulation around the stationary cylinder geometry. By using this novelty, thousand of various cases are contrived to be conducted. Scheduled experiments are estimated to constitute the infrastructure behind the Magnus effect and its control. That effort will be the light for the following step what is the method of usage in airfoils and related fields to control the outer forces.

\section{References}

[1] W. M. Swanson, "The Magnus Effect: A Summary of Investigations to Date", Journal of Basic Engineering, vol. 83, no. 3, p. 461-470, Sep. 1961.

[2] A. Lafay, "Sur l'Inversion du phenomene de Magnus", Compt. Rend, vol. 151, 1910.

[3] J. W. Maccoll, “Aerodynamics of a Spinning Sphere”, J. R. Aeronaut. Soc., vol. 32, no. 213, p. 777-798, Sep. 1928.

[4] E. Krahn, "Negative magnus force.", Journal of the Aeronautical Sciences, vol. 23, no. 4, p. 377-378, 1956.

[5] S. Taneda, "Negative Magnus effect.”, Rep. Res. Inst. Appl. Mech., vol 5, p. 123-128, 1957.

[6] T. Kray, J. Franke, and W. Frank, "Magnus effect on a rotating sphere at high Reynolds numbers", Journal of Wind Engineering and Industrial Aerodynamics, vol. 110, p. 1-9, Nov. 2012.

[7] J. Kim, H. Choi, H. Park, and J. Y. Yoo, "Inverse Magnus effect on a rotating sphere: when and why", Journal of Fluid Mechanics, vol. 754, p. R2, 2014.

[8] V. Modi, "On the moving surface boundary-layer control”, In Fluids 2000 Conference and Exhibit, p. $2238,2000$. 